\title{
Analysing the potential for application of the phase shift method in endoscopic examination of marine engines
}

\author{
Zbigniew Korczewski, Prof. \\ Gdansk University of Technology, Poland
}

\begin{abstract}

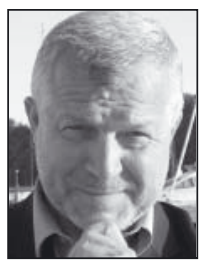

The article presents theoretical principles of image processing in digital endoscopy which makes use of a miniaturised spectral scanner "PhaseProbe" designed by General Inspection Technologies LP, the producer of the measuring videoendoscope Everest XLG3. The technology of optoelectronic 3D mapping of the examined surface, which consists in measuring the phase shift between the emitted and reflected light waves, is briefly described. The efficiency of the "PhaseProbe" based method of endoscope measurements is compared with the earlier developed methods, such as "ShadowProbe" and "LaserDots", which were used by the author in real conditions of diagnostic investigations of engines in operation in marine power plants. The processed results of the endoscope analysis of the broken gas turbine engine rotor blades which were earlier dimensioned using the inspection probes of "StereoProbe", "ShadowProbe" and "LaserDots" type and now are examined by the author with the aid of the phase shift method make a valuable complement and synthesis of the discussion presented in the article.
\end{abstract}

Key words: endoscopic diagnostics; phase shift method; marine engines

\section{INTRODUCTION}

Despite rapid technological development in measuring methods used in industrial endoscopy, precise measurement of the dimensions of the detected surface defects is still a challenging problem. New opportunities in this area have been brought by various 3D scanning techniques which consist in converting the real three-dimensional shape of the examined surface into a digital form. The theoretical principles of the phenomena of light wave diffraction and interference which accompany this mapping were worked out over 200 years ago by Thomas Young1), but only as recently as in 2010 the most modern optoelectronic devices for light emission and detection made it possible to design the measuring videoendescope Everest XLG3 by General Inspection Technologies LP. This videoendoscope makes use of the spectral scanner miniaturised to the overall dimension of the inspection probe head of an order of $6 \mathrm{~mm}[4,5,6]$. Moreover, a method has been developed which uses the results of the light wave phase shift measurement ${ }^{2}$ to create a digital 3D model of the examined surface being a precise mapping of its real geometric shape and dimensions ${ }^{3)}$.

\section{PHASE SHIFT METHOD - 3D MEASUREMENT}

A key element in the design of the inspection probe of the measuring videoendoscope Everest XLG3 in the "PhaseProbe" option is the diffraction lens mounted in the inspection probe head - Fig. 1. The design of the lens bases on a pair of symmetrically fixed diffraction gratings (2) which generate a static interference pattern having the form of halftone screens with known parameters, (i.e. gratings of dark and bright interference fringes with certain density) on the examined surface. The part of the halftone screen which is within the visual field of the image processing optics (1) is recorded on the matrix of the CCD camera as a cloud of points, the X, Y, Z coordinates of which are known. Then this

1) Thomas Young (1773-1829) - an English physicist and physiologist. Initiated the wave theory of light by stating that the light waves are transverse waves. In 1801 he discovered diffraction and interference of light waves (Young's experiment). A detailed description of these phenomena was issued by him in 1807.

2) United States Patent No.: US 7,821,649 B2.

3) In the promotional materials issued by Everest VIT in Polish, the light wave phase shift measurement method is more briefly referred to as the "3D phase method". 
pattern is analysed and converted, in the triangulation process done by the central unit of the videoendoscope, to a grid of triangles which transform the virtual image of the examined surface into its 3D equivalent. A complementary element of the "PhaseProbe" lens is the window of the standard illumination system fed with a 75-watt high-intensity discharge arc lamp (HID) having the luminous flux equal to 4300 lumen, as the measure of its brightness [3]. The resultant colour temperature of the light source ${ }^{4)}$ is approximately equal to $5000 \mathrm{~K}$. a)

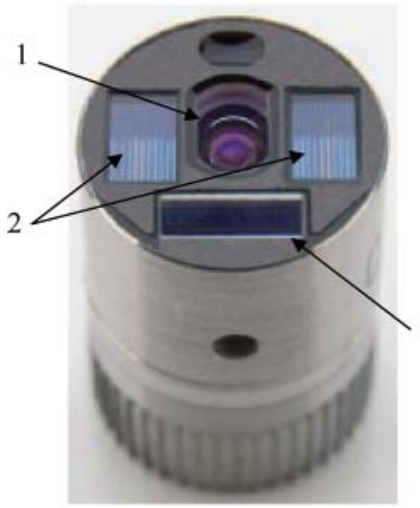

b)

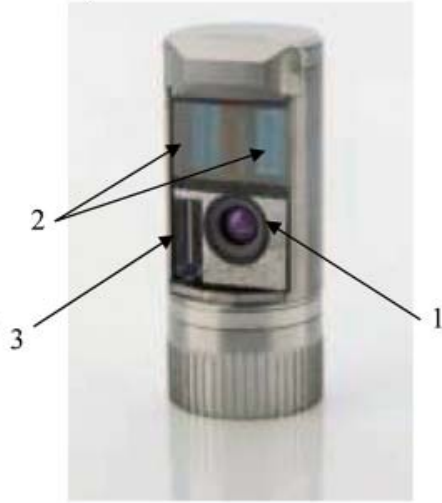

Fig. 1. Diffraction lens for measuring the phase shift with the aid of "PhaseProbe" of the videoendoscope Everest XLG3 [4]. a) straight-ahead direction of observation (field of vision angle " $F O V$ " $-105^{\circ}$, depth of field "DOF" - 8-250 mm, b) side direction of observation ("FOV" - $105^{\circ}$,

"DOF" - 7-250 mm). 1) image processing optics - CCD camera,

2) diffraction grating, 3) window of standard illumination system.

The standard illumination system of the videoendoscope works in the continuous mode only during routine observation of the examined surfaces. During the measurement the standard illumination system is automatically switched off to reach the maximal possible resolution of the interference fringe pattern generated by the diffraction gratings, which are illuminated in this time by electro-luminescence diodes (LED) radiating the precisely defined light wave length.

When the distance of the "PhaseProbe" lens from the examined surface changes, the halftone screens of diffraction fringes also undergo relevant changes, according to the schematic diagram shown in Fig. 2. Therefore based on the records and analyses of the deformation pattern of particular diffraction fringes we can conclude about qualitative and quantitative surface deformations of the examined object. Since the observed dimensions of the surface defects are not only the function of their real dimensions but also of the distance of the inspection head lens from the examined surface, its precise determination is a basic metrological problem in 3D measurements. For this purpose we can apply the phase shift method, initially described by Thomas Young - Fig. 3. Monochromatic, incoherent light waves (revealing the same length but different and timedependent phase shifts) are emitted in a sequence by the light sources LED1, LED2, LED3. These light waves undergo double diffraction: initially on the gaps which equalize the vibration phases, and then, as the monochromatic coherent waves, on the diffraction grating gaps, thus creating a pattern of bright and dark interference fringes of the same intensity on the examined body surface. The bright fringes (maxima of interference) are

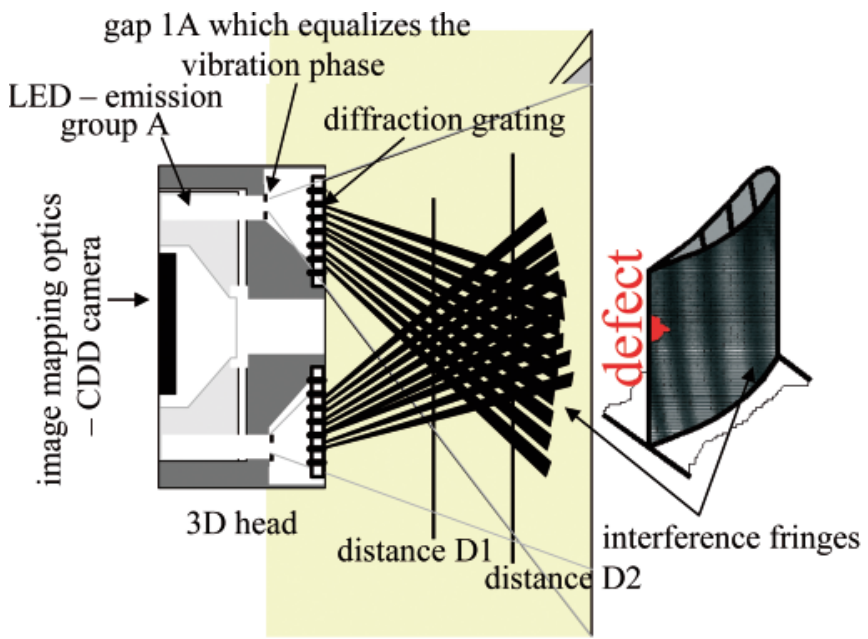

Fig. 2. Schematic diagram of the method of $3 D$ measurement with the aid of the "PhaseProbe" inspection probe diffraction lens

situated in places of highest superposition of the two interfering light waves of the same length, while the dark fringes (minima of interference) - in the place of their highest difference. We can talk about so-called constructive interference, for which the condition of the equality of the light wave optical path length difference (phase difference) and a multiple of the wave length is met, or destructive interference - when the optical path length difference of the light waves is the odd multiple of the half of the light wave length. Using the nomenclature for macroscopic quantities shown in Fig. 3, and taking into account that the distance $\mathrm{D}$ of the inspection head lens from the examined surface is much longer than the diffraction grating constant $d(D>>d)$ we can assume that the line segment $A C$ is almost perpendicular to the optical paths $r_{1}$ and $r_{2}$. That means that the angle $\mathrm{ABC}$ is almost equal to the angle PRO (denoted as $\alpha$ ). Hence the optical path length difference between the interfering light waves (being the effect of the phase shift) can be given by the following formula:

$$
\mathrm{BC}=\Delta \mathrm{r}=\mathrm{d} \cdot \sin \alpha
$$

Thus the condition for the appearance of the bright interference fringe on the examined surface is identical with the constructive interference condition:

$$
\mathrm{d} \cdot \sin \alpha=\mathrm{n} \cdot \lambda
$$

where:

$\mathrm{n}=1,2,3 \ldots-$ is the order of the successive interference fringe.

For point $\mathrm{P}$ in Fig. 3, the phase difference of the emitted light waves is equal to their four lengths.

The condition for the appearance of the static dark fringe in interference pattern is identical with the destructive interference condition:

$$
\mathrm{d} \cdot \sin \alpha=(2 \mathrm{n}+1) \cdot \lambda / 2
$$

where:

$\mathrm{n}=1,2,3 \ldots-$ is the order of the successive interference fringe.

4) The colour temperature is the method of visible light evaluation used in illumination, photography and filming. The colour temperature of the source of light is assessed by comparing its chromaticity to that of the perfectly black body. The colour temperature is usually measured in Kelvin degrees, and it is the temperature to which the perfectly black body is to be heated to obtain the given colour. Contrary to a common sense, a higher colour temperature (above 5000K), means "cool" (green-blue) colours, while a lower colour temperature (2700-3000K) means "warm" (yellow-red) colours. The "cool" light is considered better in case of tasks which need concentration [www. ModernHome.pl]. 


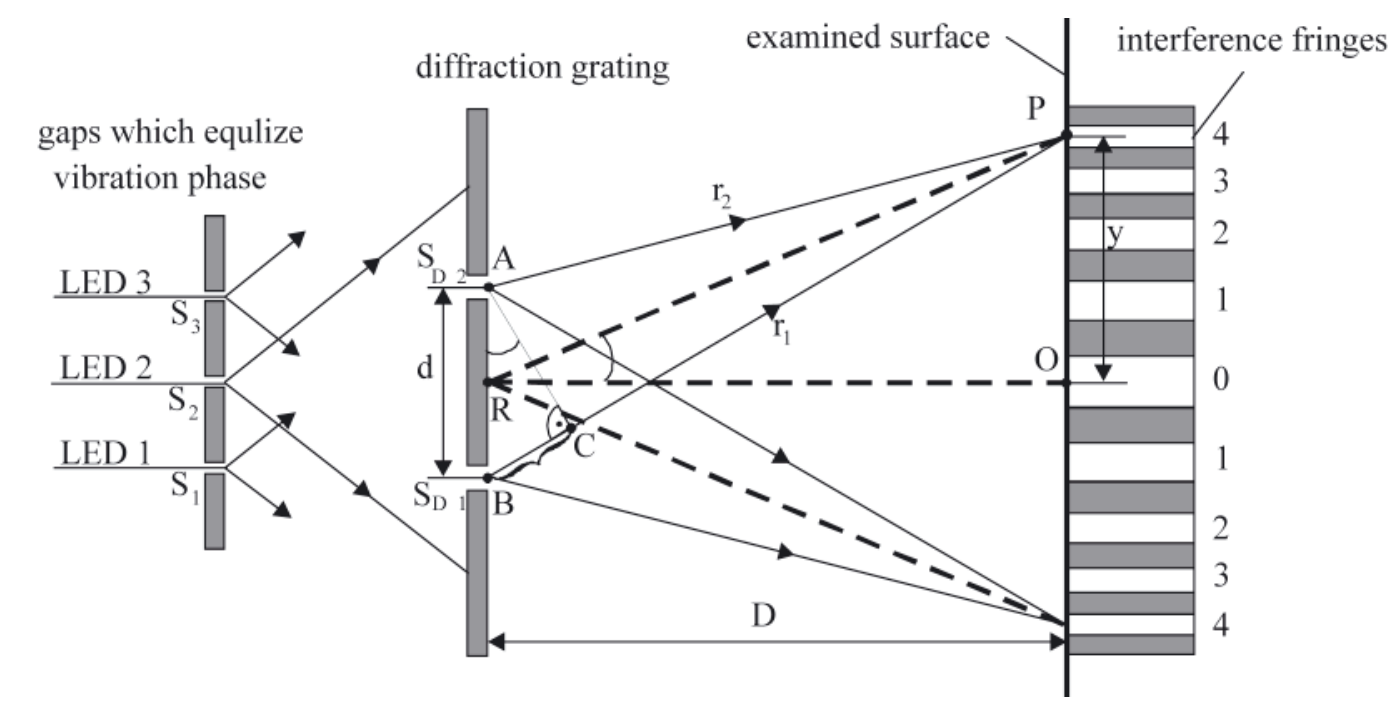

Fig. 3. Method of calculating the distance between the inspection head and the examined surface in the phase shift method. $\boldsymbol{S}_{1}, \boldsymbol{S}_{2}, \boldsymbol{S}_{3}-$ gaps which equalize the vibration phase, $\boldsymbol{S}_{D 1}, \boldsymbol{S}_{D 2} \ldots \boldsymbol{S}_{D n}$-diffraction grating gaps (for clarity of the picture, their structure is presented in part only and is overenlarged), $\boldsymbol{r}_{1}, \boldsymbol{r}_{2}$-optical paths of the light waves, $\boldsymbol{d}$-diffraction grating constant (distance between two neighbouring gaps, diaphragm width), $\boldsymbol{D}$-distance of the inspection head lens from the examined surface, $\boldsymbol{y}$-distance of the interference fringe of a given order from the zero-order fringe,

$$
0,1,2,3,4 \ldots n \text {-order of the successive interference fringe }
$$

If the dark interference fringe was expected to appear at point $\mathrm{P}$ in Fig. 4, the phase difference between the emitted light waves should be equal to the odd multiple of the half of the wave length, i.e. four and half of the wave length.

Since the light beam deflection angle $\alpha$ at which the bright fringes are created increases for each successive interference number, the value of the deflection angle sine function increases as well, thus determining the distance between particular fringes in the interference pattern. The first-order fringe is always situated opposite the diaphragm, between the gaps $d$ and is the symmetry axis of the interference pattern.

If we manage to create a stable and sharp pattern of widely distributed interference fringes on the examined surface as a result of light wave diffraction and interference, we can measure with high precision their distances from the zero-order fringe and then, having known the length $\lambda$ of the emitted light wave and the diffraction grating constant $\mathrm{d}$, calculate the distance of the videoendoscope inspection head lens from the examined surface D using the following formula:

$$
\mathrm{d} \cdot \frac{\mathrm{y}}{\sqrt{\mathrm{D}^{2}+\mathrm{y}^{2}}}=\mathrm{n} \cdot \lambda
$$

Hence:

$$
\mathrm{D}=\mathrm{y} \cdot \sqrt{\left(\frac{\mathrm{d}}{\mathrm{n} \cdot \lambda}\right)^{2}-1}
$$

Of high importance, from the technological point of view, in the production of the probe lens of the "PhaseProbe" videoendoscope is precise incision of two diffraction gratings, consisting of parallel and equidistant grooves having minimal dimensions of an order of 1-2 mm, on two glass plates using a diamond cutting edge. These grooves compose the diffraction grating diaphragms which separate its optical gaps. The width of the gap is to be approximately equal to the width of the groove and, simultaneously, to the light wave length.
The sine qua non condition for obtaining a stable pattern of the interference fringes from the diffraction grating is securing a monochromatic and coherent light source ${ }^{5)}$. The light waves emitted by the LED diodes are monochromatic, which means that they have the same wave length $\lambda$, but they are not coherent, i.e. the sinusoidal waves of the light beam do not vibrate with the same amplitude, frequency and phase, or time-independent phase difference. To solve this problem, the incoherent source of LED light generates the waves on the diffraction gratings of the "PhaseProbe" head lens via additional gaps which equalize their phases (according to the Huygens principle6), the waves which leave the diffraction grating gaps are generated by vibrating points situated on the same wave front, which means the presence of a constant phase difference between them).

To provide opportunities for complete and accurate 3D mapping of the examined surface, the phase shift method makes use of a system of two diffraction gratings, each o which is fed from three LED emission groups which emit, in a sequence, sinusoidal light waves mutually shifted by a phase angle equal to $120^{\circ}$ (this solution secures high sharpness of the static interference pattern) - Fig. 4.

This way a rapidly changing projection and recording of two patterns (half-tone screens) of interference fringes with known density take place on the examined surface. These fringes are deformed adequately to the scale of surface deformation (they deflect left when the material has piled up and right in case of material decrement). The pattern of the light reflected from the examined surface is recorded on the CCD camera matrix.

The videoendoscope processor system (CPU) identifies and localises particular fringes. Then it performs a comparison analysis of the phase shift between the known emitted light and the reflected light. The results of the calculations, performed taking additionally into account parameters of camera calibration and the angle between the direction of the projection and that of the halftone screen recording, have the

5) This condition can be only met by laser light sources.

6) Christiaan Huygens (1629 -1695) - Dutch mathematician, physicist and astronomer. In the area of physics, he performed a number of works on optics. He formulated the Huygens principle, which says about the wave propagation in the vicinity of obstacles. His experiments confirmed the theory on the wave nature of light. He also examined the phenomenon of light refraction in various materials. 
form of X, Y, Z coordinates for each camera pixel. The cloud of points created in the above manner, the number of which results from the resolution of the applied camera, makes the basis for dimensioning the detected surface defects.

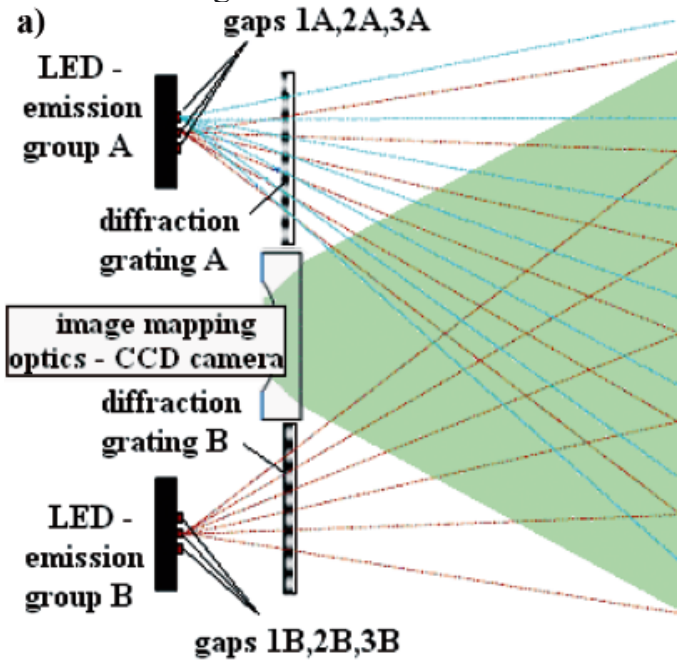

b)

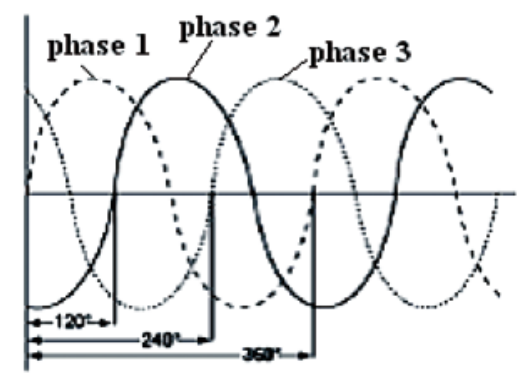

Fig. 4. Phase shift in the 3D measurement method [6]. a) interference pattern generated by the inspection head lens, b) phase shift pattern of the sinusoidal light waves emitted by three emitting groups LED 1,2 and 3 and the diffraction gratings , $A$ " and , $B$ ".

\section{ADVANTAGES AND DISADVANTAGES OF THE 3D MEASUREMENT METHOD}

The videoendoscope Everest XLG3 is equipped with a 3D measurement probe bearing the name of "PhaseProbe" which is a "flexible three-dimensional eye" of the operator. These borescopes reveal numerous advantages, which are the reason why they are in more and more frequent use. The basic advantage of the 3D measurement technology with respect to older measuring methods, such as "StereoProbe", "ShadowProbe", and "LaserDots" for instance, is its ergonomics. Switching from the standard (qualitative) measurement mode to the measuring (quantitative) mode does not require withdrawing the inspection probe from the inside of the examined object and changing the optic lens. Apart from complicated manual work to be done to change the lens, a big problem in those cases was finding again the earlier detected surface defect, the more so that the measuring lens reveal, as a rule, low quality of image processing of the examined surface observed from a larger distance. For instance, the depth of field for "ShadowProbe" lens is within 7-30 mm, while for "PhaseProbe" it ranges between 7-250 mm!

On the other hand, "PhaseProbe" is very sensitive to image movements (vibration) and light reflection from highly reflective surfaces. The measurement cannot be performed, in practice, when the operator does not manage to keep the tip of a flexible and a number of meters long probe still and situated at a proper angle for at least two seconds. It is extremely difficult to reach this state, even if a special probe rigidiser with a tube gripper is used for this purpose [2,3]. From the practical point of view it is a serious disadvantage of the "PhaseProbe" based $3 \mathrm{D}$ measurement method and it should be eliminated in the future by its inventors.

Taking into account high cost of purchase of the measuring set, which, despite the decreasing trend, still remains at the level of 40-50 thousand Euro, each time a decision about its purchase should have strong rational background.

When discussing possible areas of application of the measuring videoendoscope with "PhaseProbe" in marine engine diagnostics, working spaces should be mentioned for which surface wear is decisive for the efficiency of the energy conversion processes taking place in the engine. Tasks to be done in those cases include precise determination of parameters characterising the roughness of the surfaces composing the borders of the working space. This should be done over a relatively large area, which from the point of view of endoscope diagnostics requires its full numerical mapping and dimensioning. In those cases the quantitative measure of wear of the examined surface is the arithmetic mean deviation of its profile from the average line determined along a normalised elementary line segment, or the roughness height calculated using ten points of this profile.

The research experience gained in the past by the author suggests that the phase shift method can be efficiently used for diagnosing the following constructional elements:

1. For working spaces of piston engines:

a) cylinder bearing surface - honing grooves,

b) valve seats - valve set faces, wear thresholds on valve heads,

c) air and exhaust gas flow ducts - shape and geometric dimensions, active flow sections, condition of inner surfaces,

d) turbo compressor rotor assembly - shape and geometric dimensions of blades, condition of blade profile surfaces and inter-blade passages;

2. For flow parts of turbine engines:

a) rotor assemblies of compressors and turbines - shape and geometric dimensions of stator and rotor blades, condition of blade profile surfaces and inter-blade passages,

b) combustion chamber - shape and geometric dimensions of blade systems in flame tube swirl vanes, condition of inner and outer surfaces of flame tubes.

The measuring methods of "Stereo", "Shadow" and/or "LaserDots" type provide opportunities for digital processing of stereoscope effects, which makes it possible to dimension the observed objects in such a way that they give an impression of quasi three-dimensionality with its depth, massiveness and mutual distribution. Unfortunately, their application to full (sufficiently detailed), three-dimensional mapping of larger surfaces is very limited for the following reasons:

1. The "Stereo" method: the 3D measurement bases on precise correlation of two identical characteristic points of the left and right view of the dispersed stereoscope image [4]. A problem can arise when no clear characteristic points can be found on the examined surface. The second limitation is remarkable labour consumption of the measurement which results from small angles of the visual field and depths of field of the used "Stereo" type lens, which are equal to: „FOV" $-45 \div 60^{\circ}$, „DOF" $-2 \div 80 \mathrm{~mm}$. This prolongs the time of the measurements necessary for full three-dimensional mapping of the entire examined surface. For these reasons the "Stereo" method is more efficient in cases when only a limited number of surface points is required for measuring basic dimensions of the detected defects. 
2. The "LaserDots" method: the 3D measurement consists in projecting a matrix of markers composed of a number (49, for instance) of laser beam points on the examined surface and numerical recording of their shift to the left or right of the screen as the result of surface profile changes [2]. Since the areas situated between the matrix markers cannot be interpolated in this way, any surface changes taking place in these areas cannot be dimensioned either. As a consequence, the 3D mapping of the examined surface is only limited to laser marker points, which does not secure the required resolution of the measurement.

3. The "Shadow" method has a form of single-fringe scanning and brings useful three-dimensional information only with respect to one plane of the examined surface profile within a very limited area, as a result of optical limitations of the applied "Shadow" type lens (,FOV” - 50 , „DOF” $-7 \div 30 \mathrm{~mm}$ ). An additional difficulty in the realisation of the 3D measurement with the aid of the "Shadow" method is the need for very precise linear positioning of the fringe with respect to the examined surface, which requires some experience. If the surface is not ideally flat, or the inspection probe lens is not directed perpendicularly, then a relatively large error can be recorded in the measurements which require referring the baseline to the points situated off the line, the line-point distance measurement for instance. For these reasons, and bearing in mind the fact that, like in the "Stereo" method, a huge-capacity processor is to be installed in the videoendoscope to solve equations of the mathematical model describing the $3 \mathrm{D}$ profile of the examined surface in this technique, the application of the "Shadow" method is rather limited to only dimensioning the detected surface defects [2].

\section{D MEASUREMENT TECHNOLOGY}

The endoscopic examination of inner spaces of the engine using the "PhaseProbe" of the videoendoscope Everest XLG37) can be realised in the standard mode or the measuring mode without change of inspection head lens. The standard examination is oriented on general qualitative evaluation of the technical state of a constructional element in the engine, along with the search for and identification of failures of surface defects. If any change in the constructional structure of the examined elements is identified, an attempt is to be made to evaluate it in quantitative way using the measuring mode for this purpose. As already mentioned, it does not require any additional actions concerning the operation of the inspection probe, except moving its lens as close as possible to the examined surface, while preserving at the same time a sufficiently large depth of field (like in the measurements performed in the "ShadowProbe" and "StereoProbe" options) and an optimal visual angle $\left(45^{\circ}\right.$ is the recommended angle in case of the examination of shining or spotty surfaces). A remarkable advantage of the software of the videoendoscope equipped with "PhaseProbe" is continuous analysis and adjustment of the distance between the measuring lens and the examined surface. During the measurement of the phase shift, a range-finder strip is displayed on the LCD monitor of the video-probe handset ( 1 in Fig. 6a). Exceeding the measuring range is signalled by a single red strip, which means that we should not make attempt to measure. When the distance of the lens from the examined surface decreases, it results in the increasing number of the displayed strips, which, what is more, change the colour to green, which means that the measurement can be done. Scanning, started by pressing the push-button Enter on the manual panel, initiates the process of fast (lasting about 1-2 seconds) and multiple recording of the digital image. This is the crucial action of the technology of 3D measurement (as mentioned above), which lasts until the message "Processing" is displayed on the monitor screen. After the measurement is completed, the distance of the lens from the examined surface (at the point of the active cursor) is displayed on the monitor as the MTD (Maximum Target Distance) index. Unlike the "StereoProbe" and "ShadowProbe" methods, the phase shift method does not require additional correction of the measurement depending on this distance ("Accuracy Index"). Further processing of the recorded image is done using a specialist software, GE Inspection Manager v.06 ${ }^{8}$, see Fig. 5, after completing the endoscope examination.

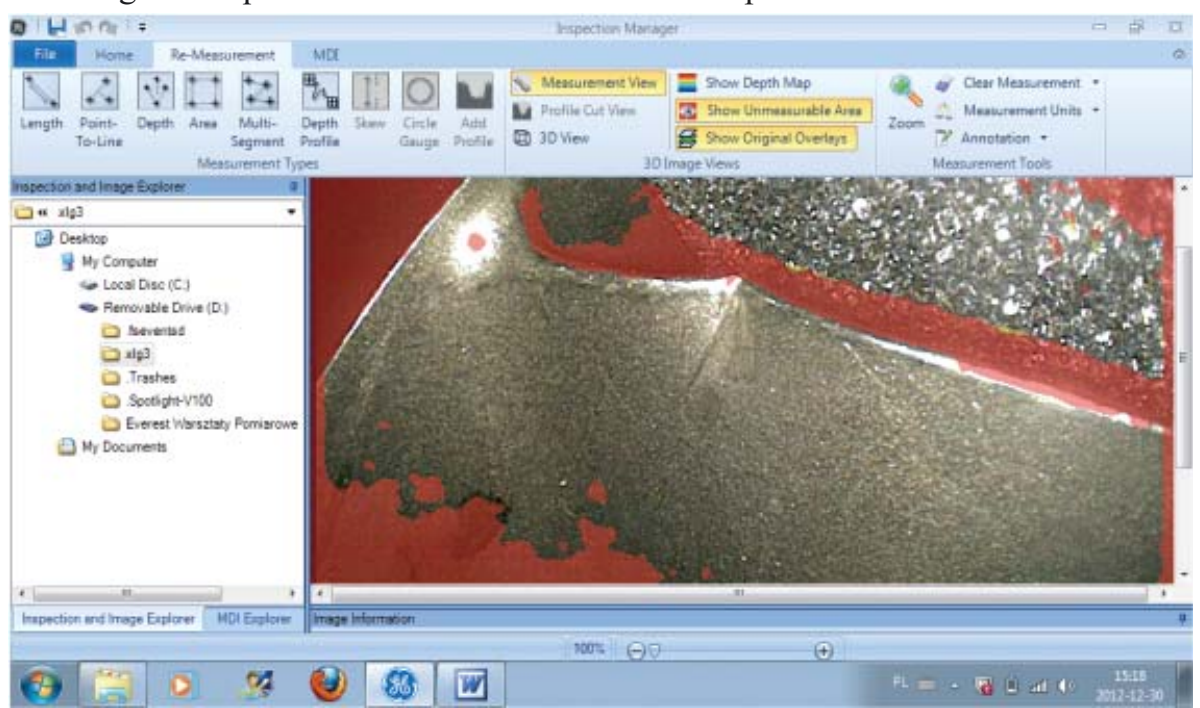

Fig. 5. View of the computer screen after starting the programme GE Inspection Manager v.06 in the "Measurement area" mode (image parts which are not accessible for measurement as excessively distant, dark or screened by light reflection are marked red)

7) Due to volume limitations of the present article, it does not include detailed constructional and parametric characteristics of the videoendoscope Everest XLG3. This information can be found on internet pages of General Inspection Technologies LP and dealers of Everest VIT endoscope equipment. Moreover, detailed descriptions of the methodology of the endoscope based diagnostic investigations of marine engines, which are also omitted here, can be found in numerous earlier publications by the author.

8) By courtesy of "Everest VIT GmbH Poland". 
In the "PhaseProbe" method the following measuring options are available:

- length,

- multi-segment length, length of a broken line (perimeter),

- distance of a point from the straight basic line,

- depth (convexity),

- diameter of the area marked by a circular rule,

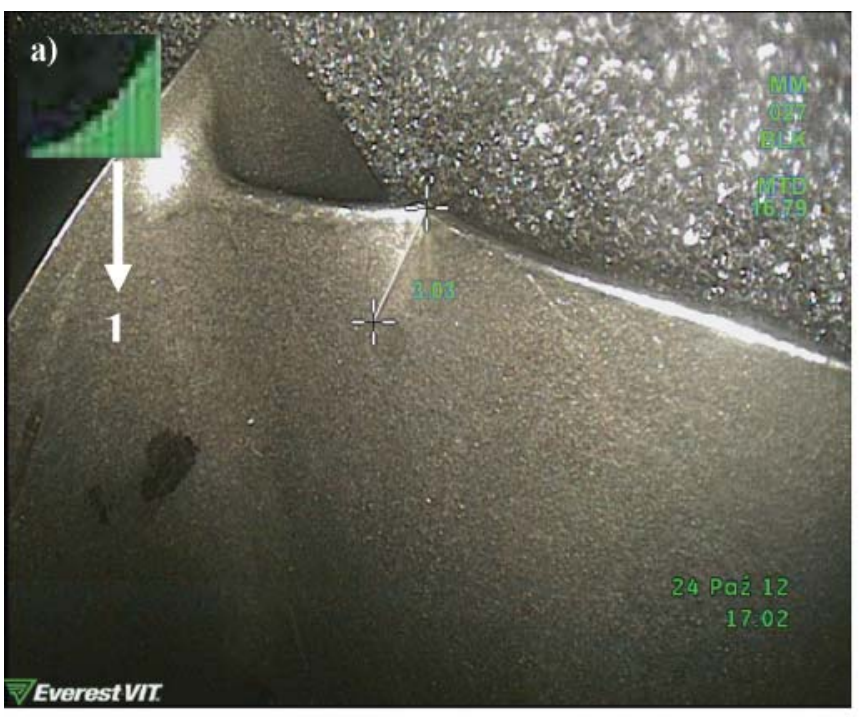

c)

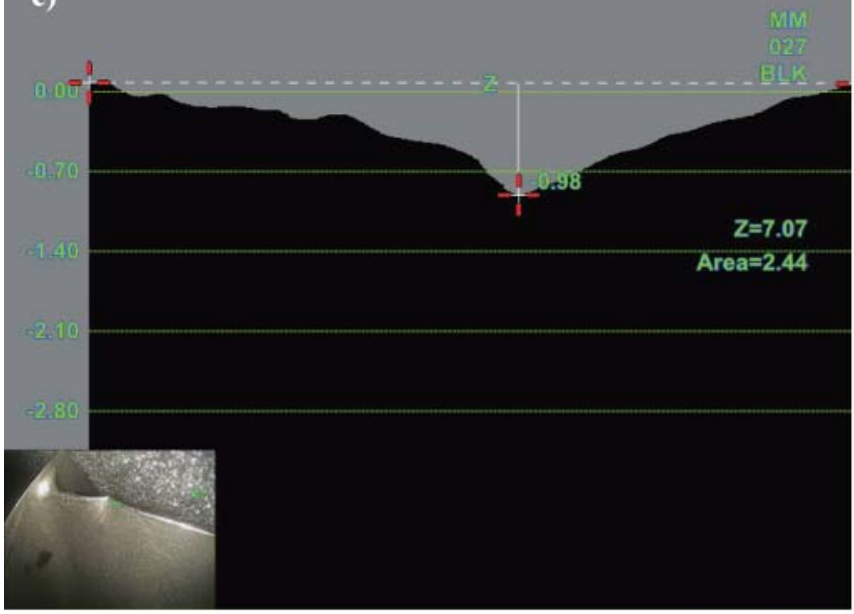

e)

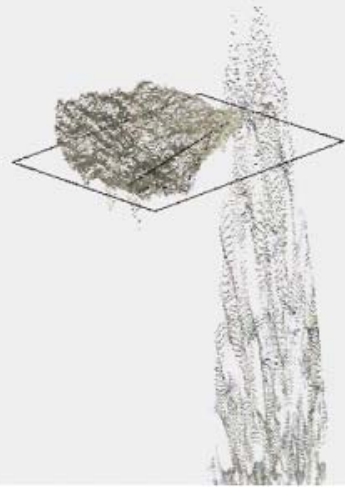

- profile and profile map of the examined surface,

as well as the 3D view of the cloud of the measuring points which map the examined surface, with options of its rotation with respect to $\mathrm{X}, \mathrm{Y}$ and $\mathrm{Z}$ axis.

Figures 6 and 7 show the processed results of the endoscope examination of the turbine engine rotor blades using "PhaseProbe" and the phase shift method. The surface defects

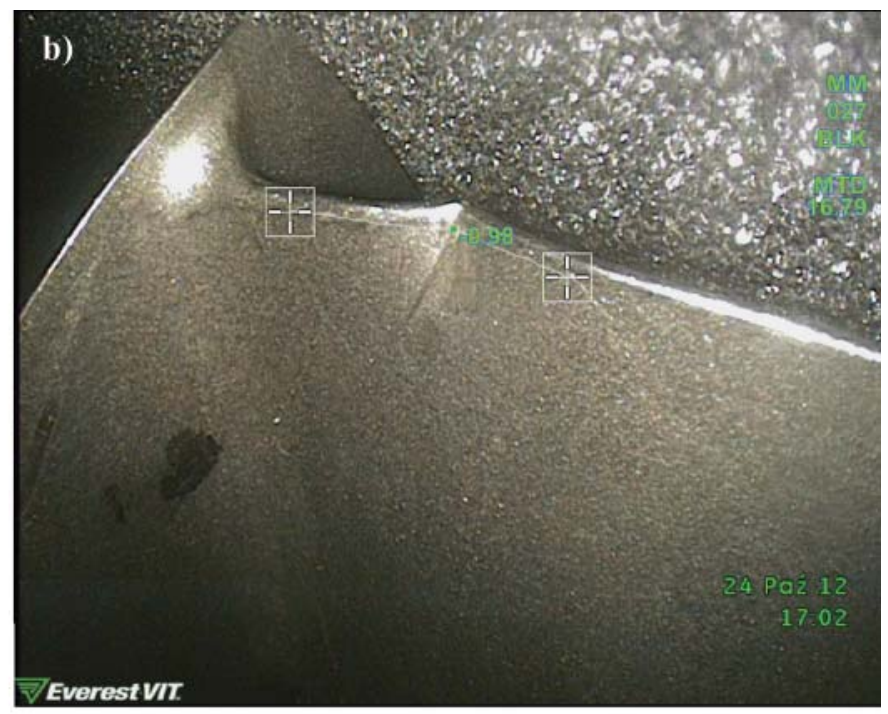

d)
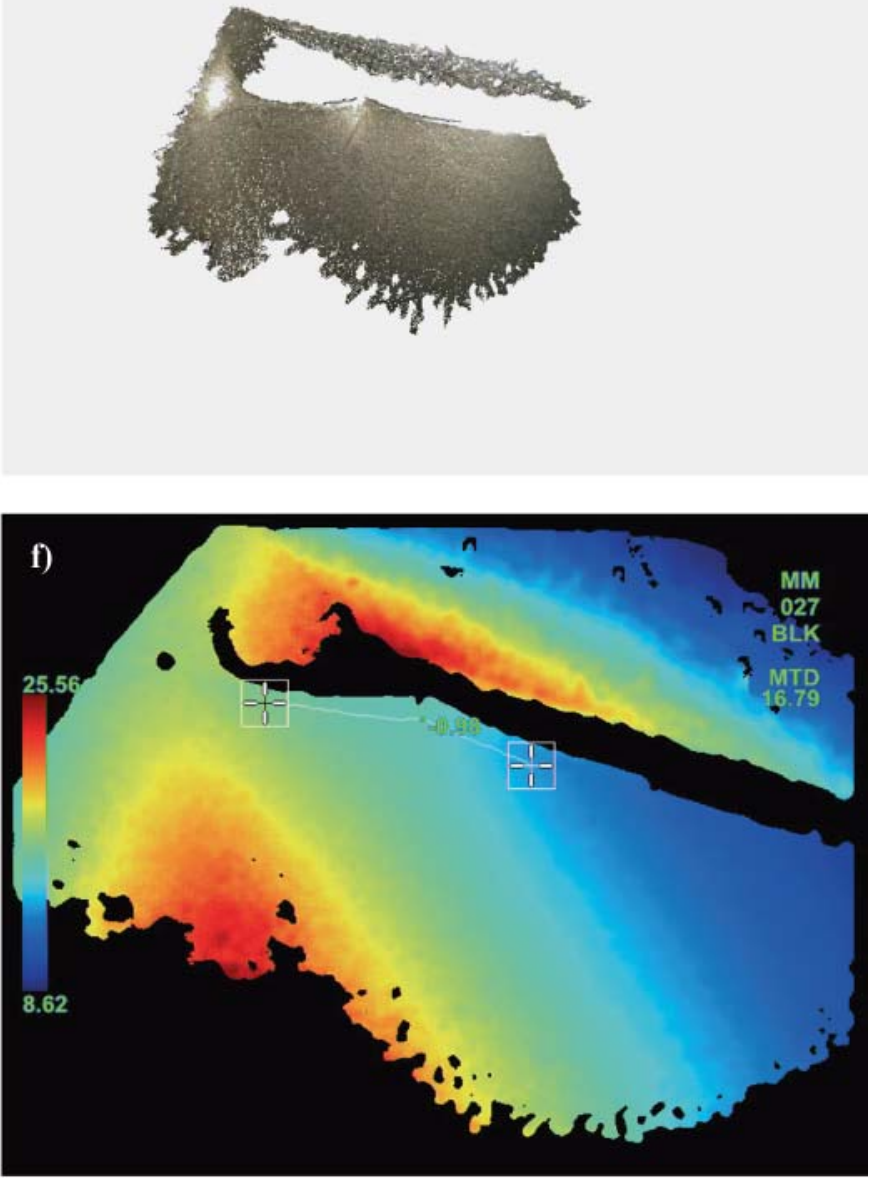

Fig. 6. Results of the $3 D$ measurement of the indentation on the turbine engine rotor blade trailing edge performed with the aid of the phase shift method. a) $1 \mathrm{D}$ measurement of indentation length $-3.03 \mathrm{~mm}$, at MTD index - $16.79 \mathrm{~mm}$, b) measurement of indentation profile - $0.98 \mathrm{~mm}$, at MTD index - $16.79 \mathrm{~mm}$, c) indentation profile sector $-0.98 \mathrm{~mm}$, at MTD index $-16.79 \mathrm{~mm}$, distance between cursors $Z=7.07 \mathrm{~mm}$, d) $3 D$ view of the cloud of measuring points, e) $3 D$ view of the cloud of points with the reference plane, $f$ ) dimensioned map of surface deformation profile 

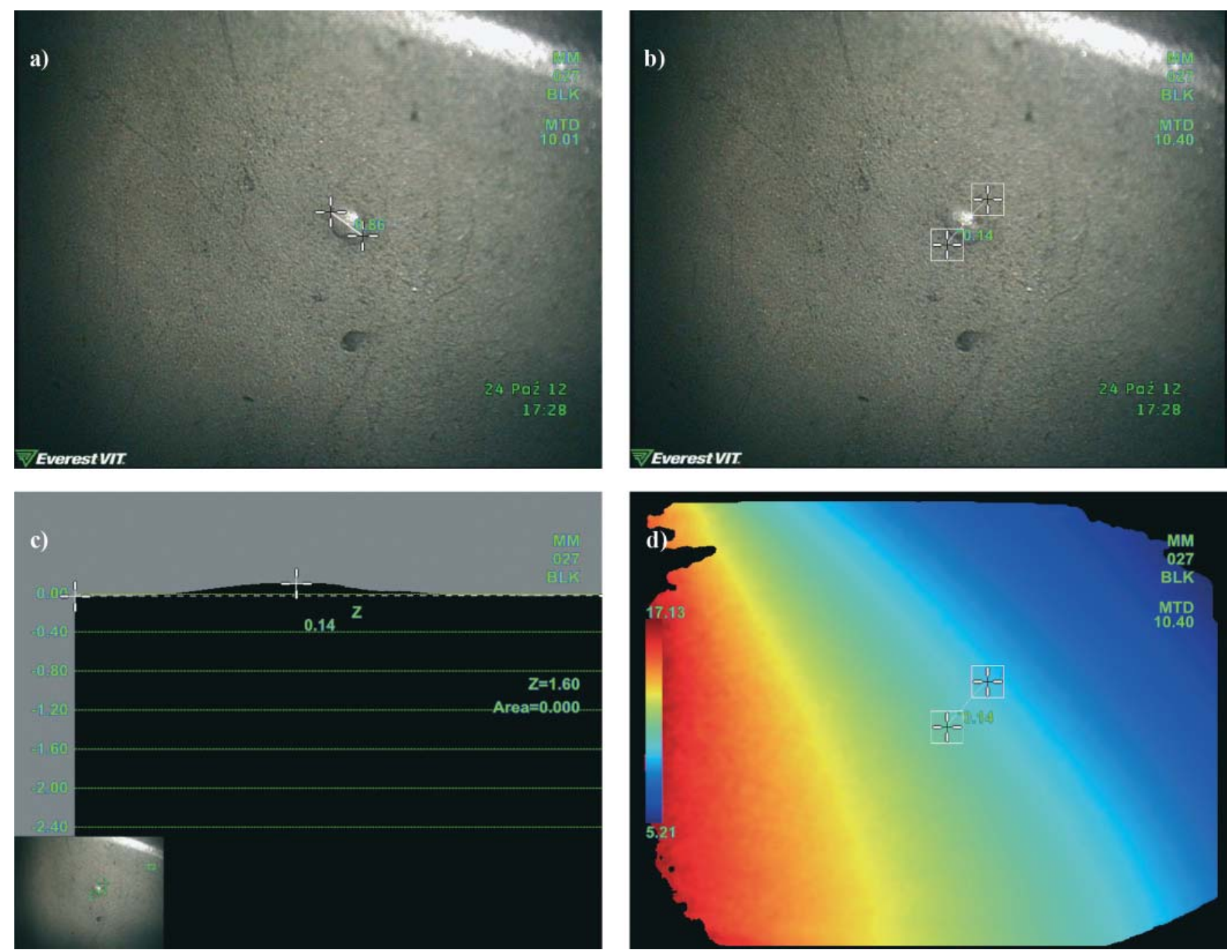

Fig. 7. Results of the 3D measurement of local material deposit ("eczema") in the turbine engine rotor blade trough performed with the aid of the phase shift method. a) $1 D$ measurement of deposit diameter - $0.86 \mathrm{~mm}$, at MTD index $-10.01 \mathrm{~mm}$, b) measurement of deposit profile $-0.14 \mathrm{~mm}$, at MTD index $-10.40 \mathrm{~mm}, \boldsymbol{c}$ ) deposit profile sector, distance between cursors $Z=1.6 \mathrm{~mm}, \boldsymbol{d})$ dimensioned map of surface deformation profile

selected for $3 \mathrm{D}$ processing and dimensional analysis of the recorded numerical images had been earlier examined with the aid of the measuring probes "StereoProbe", "ShadowProbe" and "LaserDots" [2]. Figures 6a and 7a show the results of standard dimensioning of, respectively, the length of indentation and point material deposit on the examines turbine blade. The examination was performed at rather large values of the MTD index: 16,79 $\mathrm{mm}$ and 10,01 $\mathrm{mm}$. These results are very similar to those obtained in the past using other measuring methods. But completely new metrological capabilities have been brought by the 2D measurement of surface deformation profile, Figs. $6 \mathrm{~b}, 6 \mathrm{c}, 7 \mathrm{~b}$ and $7 \mathrm{c}$, the three-dimensional cloud of measuring points, Figs $6 \mathrm{~d}$ and $6 \mathrm{e}$, and the dimensional map of surface deformation profile, Figs. $6 \mathrm{f}$ and $7 \mathrm{~d}$. A possibility to rotate the digital model of the examined surface which precisely models its real shape and geometrical dimensions makes the process of diagnostic inference easier and more efficient, and remarkably increases the reliability of the formulated diagnosis.

\section{FINAL REMARKS AND CONCLUSIONS}

A basic condition for formulating a reliable endoscope diagnosis of the technical state of working surfaces in a marine engine is an opportunity to perform not only qualitative but also quantitative assessment of the detected surface defects. New perspectives in this area are brought by digital endoscopy. Numerical image analysers cooperating with the measuring heads of "StereoProbe, "ShadowProbe", "LaserDots" and here described "PhaseProbe" type provide opportunities for numerical processing of stereoscopic effects which in turn makes it possible to dimension the observed images in such a way that they give an impression of quasi three-dimensionality, with its depth, massiveness, and mutual distribution.

Recent years show that further development of the endoscopic diagnostics of marine engines is absolutely determined by increasing technical capabilities of the more and more perfect and faultless measuring equipment. Precise and ergonomically designed digital endoscopes open new prospects for developing the diagnostic knowledge on the kinetics of working space wear in engines in operation, leaving less and less space for traditionally used optical endoscopes [1]. However, there is one sine qua non condition: the diagnostician should study in detail technical capabilities of the owned measuring videoendoscope and be able to use them effectively in the environmental conditions in which the diagnostic examination is performed. He also should avoid operating mistakes which would lead not only to the decrease of the life time and reliability of the very expensive measuring endoscope equipment, but also to worsened reliability of the diagnosis of the examined engine. 


\section{REFERENCES}

1. Hlebowicz J.: Industrial endoscopy (in Polish). Biuro Gamma. Warsaw 2000.

2. Korczewski Z.: Endoscopy of marine engines (in Polish). AMW, Gdynia 2008.

3. Korczewski Z.: Analysing the ability to identify the technical and energetic state of a marine main engine piston-crankshaft system during its operation taking into account diagnostic parameters measured with the aid of methods used in endoscopic diagnostics (in Polish). Report prepared within the framework of the research project financed by z MNiSW No N509 494638 and entitled : „Decision making based control of operation of piston-crankshaft systems in marine main engines of sea-going vessels performed with the aid of technical diagnostics and taking into account the safety and environment protection." Gdansk University of Technology, 2010.

4. General Electric Company Inspection Technologies. The Everest XLG3 VideoProbe System. Operation Instruction. USA 2011.
5. General Electric Company Inspection Technologies. The Everest XLG3 VideoProbe System. Manual Guide. USA 2011.

6. VideoProbe System - GE Measurement \& Control. USA 2011.

\section{CONTACT WITH THE AUTHOR}

Zbigniew Korczewski, Prof. Faculty of Ocean Engineering and Ship Technology

Chair of Marine and Land Power Plants

Gdansk University of Technology

Narutowicza 11/12

80-233 Gdansk, POLAND

e-mail: sek4oce@pg.gda.pl

phone/fax: +48 583472181 